\title{
A MECHANISTIC MODEL FOR THE ELECTROCHEMICAL FACETTING OF METALS WITH DEVELOPMENT OF PREFERRED CRYSTALLOGRAPHIC ORIENTATIONS
}

\author{
E. V. Albano*, H. O. Martin ${ }^{\dagger}$ and A. J. Arvia* $\ddagger$
}

\begin{abstract}
* Instituto de Investigaciones Fisicoquímicas Teóricas y Aplicadas. Facultad de Ciencias Exactas, Universidad Nacional de La Plata, Sucursal 4, Casilla de Correo 16, (1900) La Plata, Argentina

†Laboratorio de Física Teórica, Facultad de Ciencias Exactas, Universidad Nacional de La Plata, Casilla de Correo 67, (1900) La Plata, Argentina
\end{abstract}

(Received 27 May 1987; in revised form 17 August 1987)

\begin{abstract}
A model for the development of surface profiles of face-centred cubic metals which can be related to the electrochemical facetting with preferred, oriented crystallographic planes, is proposed and simulated by means of the Monte Carlo method. Successive cycles of selective electrodissolution and electrodeposition under a periodic potential are simulated through the withdrawal and attachment of particles to the metal profile according to specified rules which are supported by experimental observations. The model is applied to the development of two different crystallographic faces starting from either perfectly-ordered void-free profiles (single crystal approach) or a rough profile with defects in the bulk (polycrystal approach). The simulation results are in qualitative agreement with electrochemical facetting data, scanning electron microscopy and scanning tunneling microscopy images of various face-centred cubic metals.
\end{abstract}

\section{INTRODUCTION}

The development of electrochemical facetting with preferred oriented crystallographic planes of facedcentred cubic(fec) metals such as platinum, gold, rhodium and palladium can be accomplished through cycles of electrodissolution and electrodeposition of the metal under particular conditions involving a pulsating diffusion-boundary layer of average thickness of the order of $10^{3} \mathrm{~nm}$. This small value can be achieved by applying to the metal in contact with an acid electrolyte either a periodic potential at a frequency greater than a few $\mathrm{kHz}$ or a $d c$ potential $[1-4]$ with periodic modulation at a frequency also exceeding a few $\mathrm{kHz}$. The upper and lower potential limits of the periodic potential are chosen to be more positive and more negative, respectively, than the potential of the corresponding metal-metal ion reversible electrode. Therefore, the upper potential influences the rate of the electrodissolution reaction, whereas the lower one affects the rate of the electrodeposition reaction. In addition, both reactions can also be coupled to adsorption-desorption processes, which in turn have a marked effect on the type of crystallographic face resulting through electrochemical fucetting. The kinetics of electrochemical facetting implies a complex mechanism which is not yet fully understood, although it appears to be closely related to that of the metal-metal ion electrode reactions under stationary conditions. At present there is evidence that electrochemical facetting, at least for fcc metals, follows a common reaction pathway [4], and from this one expects that the global process can be explained in terms of a common physical mechan-

¥Author to whom correspondence should be addressed. ism comprising cyclic withdrawal and attachment of atoms at the metal surface during the application of the fast perturbing potential. One promising way of approaching the problem which circumvent the cumbersome situation arising from both theoretical and experimental investigations on the electrochemical facetting mechanism is Monte Carlo simulation, which has been frequently used to study the growth kinetics of new phases in electrochemical systems [5-8].

In this work Monte Carlo simulation is applied to the development of two types of profiles by starting from three different initial profiles, namely a straightline profile (ideal two-dimentional (2-D) single crystal approach), a simple saw-tooth profile (2-D facetted approach) and an irregular rough profile (2-D polycrystal approach). Monte Carlo simulation results on the electrochernical facetting of fcc metals can be qualitatively correlated to various experimental results previously obtained through voltammetry, scanning electron microscopy and scanning tunneling microscopy.

\section{THE MODEL}

The Monte Carlo simulation deals with the structural modifications of the starting electrode induced by successive electrodissolution-electrodeposition cycles. The starting electrode is simulated by a square lattice of size $L_{1}$ (i-axis) $\times L_{2}$ ( $j$-axis), which corresponds to a planar section of the 3-D configuration (Fig. 1). Periodic boundary conditions on the $j$-axis have been imposed to avoid finite-size effects. In order to account for the surface roughness characteristics of a real polycrystalline electrode, the starting electrode has been filled at random with a linear concentration gradient along the $i$-axis. Each site of the $i$-th layer has 


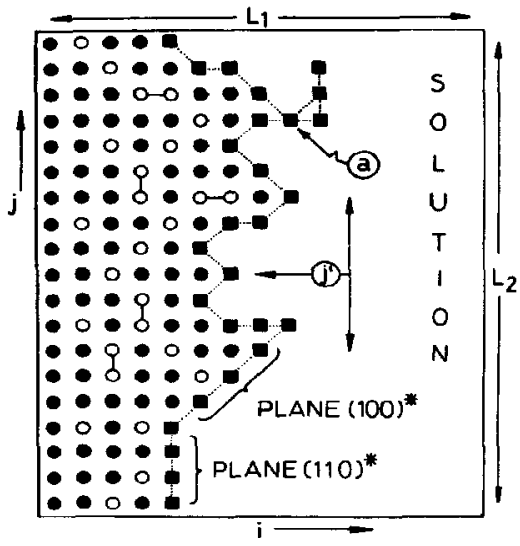

Fig. 1. Section of the electrode used in the simulation. Occupied sites, 1 profile particles in contact with the solution at the right side of the figure, 0 vacancies, $0-0$ divacancies, etc. The dotted line indicates the electrode profile. The particle (a) can not be dissolved because it would cause the disaggregation of more than one particle. The double arrow indicates the diffusion interval with $L_{D}=3$ centred in the $j^{\prime}$ coordinate which has been chosen at random. The (110)\# and (100) \# profiles are also shown in the figure. The minimum number of particles admitted for defining each plane is equal to 3 . The profile of the figure has 11 and 13 particles liying in the $(100) \#$ and the $(110)$ \# profile, respectively.

an occupation probability $P(i),(1-P(i)$ to be empty). $P(i)$ is given by

$P(i)= \begin{cases}\frac{\left(L_{3}-p\right)+(p-1) i}{\left(L_{3}-1\right)} & \text { for } 1 \leqslant i \leqslant L_{3} \\ 0 & \text { for } L_{3}<i \leqslant L_{1},\end{cases}$

that is the starting electrode is simulated by assuming full occupancy in the first layer $(P(i=1)=1)$, and $P(i$ $\left.=L_{3}\right)=p$, where $\left.L_{3}<L_{1}\right)$ and $p<1$ are adjustable parameters.

Most of the simulations were carried out by keeping $L_{1}=51, L_{2}=101$ and $L_{3}=21$, and by using $p=0.6$ or $p=0.8$. Both the case $p=1$ which corresponds to a starting electrode profile which can be taken as the $x-y$ projection of a (110) face of a fcc crystal (Fig. 1) and the case in which the starting electrode has a periodic array of 2-D projected (100) faces have also been simulated (see section III) to check the influence of the starting electrode configuration on the final results. Since only nearest-neighbour bonds between particles are considered, the starting electrode is composed of those particles which are bonded to the first layer $(i=1)$ directly or by means of bonds between adjacent particles. That is, after filling the lattice for the first time, particles and clusters which do not fulfill the above-mentioned condition are removed before starting the actual electrochemical facetting cycles. The electrolyte solution is represented by the ensemble of empty sites connected by means of nearest-neighbour bonds between empty sites to the last layer $\left(i=L_{1}\right)$. The electrode profile is composed of by those particles which are in contact with the electrolyte solution by at least one nearest-neighbour contact. For the rough case, this profile (Fig. 1) involves, among others, particles lying in an $x-y$ projected (110) plane of the fcc crystal [henceforth (110)\#], particles lying in the $\boldsymbol{x}-\boldsymbol{y}$ projected (100) plane [henceforth (100)\#] and particles at certain sites where its dissolution would imply the detachment of more than one particle in a single event. Most of the points in the lattice (elcctrode) are occupied while others, as a consequence of the imposed concentration gradient, are voids (ie bulk defects such as vacancies, divacancies, etc.) (Fig. 1).

Let us define the profile roughness, $S$, by the ratio:

$$
S=l / L_{2},
$$

where $l$ is the length of the electrode profile in multiples of the nearest-neighbour contact distance, and $L_{2}$ represents the length of the perfect (110) \# (Fig. 1). For the starting electrode configuration typical values are $S_{R}=2.7 \pm 0.4$ and $S_{R}-1.3 \pm 0.4$ for $p=0.6$ and $p=0.8$, respectively. These figures are similar to those usually obtained for mechanically polished fcc metal surfaces $[9,10]$.

In the experiments, the periodic potential used for developing the electrochemical facetting with a preferred, oriented crystallographic face is a symmetric, square-wave periodic potential whose upper and lower potential limits are positive and negative, respectively, with respect to the reversible potential of the metal-metal-ion electrode. The choice of this periodicpotential signal implies that both electrochemical processes undergo under constant-potential (fixed energy) conditions. Furthermore, the half-period of the symmetric periodic potential should be compatible with the diffusion length of electrodepositing particles. To complete the physical description of the model one must remember that metal ions exhibit different coordination numbers according to their position (ie face, step, border, hole, kink, etc.) in the fcc crystal latticc. The greatest coordination number for ions in the bulk of the crystal is twelve and, at the other extreme, metal ions in solution are coordinated to fix water molecules.

The dissolution rules selected for the Monte Carlo simulation are the following. The probability, $P_{w}$, for the withdrawal of a particle previously selected at random from the starting electrode profile depends on the number, $N$, of nearest-neighbour bonds with other particles in the lattice, and is given by:

$$
P_{W}= \begin{cases}1, & \text { if } N=1 \\ p_{1}, & \text { if } N=2 \\ p_{2}, & \text { if } N=3\end{cases}
$$

The withdrawal of particles from the electrode profile takes place as a one-by-one process and is forbidden if it implies the simultaneous detachment of two or more particles, as would be the case of particle " $a$ " in Fig. 1. In this case the particle remains at its original position. After a successful dissolution event, the electrode profile is calculated before the new trial is commenced. Each dissolution half-cycle is finished after $C \times L_{2}$ trials, where $C$ is an input parameter of the model which allows one to vary the fraction of particles constituting the profile which is involved in each dissolution-deposition cycle. In the examples discussed in this work (section III), the ratio between the number of particles dissolved during each cycle and the 
total number of particles at the profile is usually lower than 0.3 for $0.6<p<1, C=1$, and $p_{1}=p_{2}=0.2$. That is, for one half-cycle, only a fraction of the number of particles lying at the profile in contact with the solution are able to participate in the process successfully.

It is well established that under low-frequency ( $\sim \mathrm{Hz}$ ) potential-cycling of platinum electrodes in acids, covering the potential range of $\mathrm{H}$ - and $\mathrm{O}$ adatom monolayers, each scan implies a net electrodissolution of about $1 \%$ or less, of the monolayer[9-12]. As the frequency of the periodic potential increases the net amount of electrodissolved ions, as determined by analysis in solution, decreases to practically zero for frequencies of the order of $1 \mathrm{kHz}$ [3]. Therefore, under electrochemical facetting conditions, the number of particles electrodissolved during the anodic half-cycle can be completely electrodeposited during the cathodic half-cycle.

The adsorbed species (ions, water and foreign molecules) definitely influences the electrochemical reactions under stationary and non-stationary conditions. In electrochemical facetting as the adsorbed species affects the anodic and cathodic reactions in a different way, its presence assists the development of certain preferred crystallographic faces and multiple step-like surface structures. For this reason the lower potential limit of the periodic potential plays a significant role in the electrochemical facetting of platinum $[1-3,13,14]$ and rhodium $[4]$.

Therefore, in the model the deposition rules of all particles detached during the dissolution half-cycle are set as follows:

(i) A $j^{\prime}$ coordinate is selected at random between $1 \leqslant j^{\prime} \leqslant L_{2}$

(ii) To account for the diffusion of the particle prior to its attachment at a fixed site, all sites available on the surface for this process within a diffusion interval $j^{\prime}-L_{D} \leqslant j \leqslant j^{\prime}+L_{D}$ (Fig. 1 ) are checked initially. After that, the particle is deposited on the most stable site, as it is discussed in (iv). The parameter $L_{D}$ is a rough measure of the number of jumps in $n-n$ distance units that the particle has to perform before deposition. This process can be thought of as the relaxation of the particle on the profile stabilizing it at the lowest potential-energy site available within the diffusion interval.

(iii) After each single-deposition event the electrode profile is recalculated and the process continues through step (i). Each Monte Carlo simulation cycle concludes when all dissolved particles have been deposited. Afterwards, the simulation continues through successive dissolution-deposition cycles to accomplish the preset number of cycles. It should be mentioned that, due to the assumptions made in the model, a given number of Monte Carlo cycles does not necessarily coincide with the same number of potential cycles applied to the real electrode surface but, in principle, it is reasonable to expect a certain proportionality between them which makes possible a qualitative comparison between the Monte Carlo simulations and the experimental results.

(iv) Otherwise, depending on the type of profile being developed the following attachment rules are established:

(a) For the development of (110) \# the initial attach- ment of the particle undergoes at a new site involving three nearest-neighbour bonds between the attaching particle and those remaining at the electrode (Fig. 2). When the number of those sites within the diffusion interval is greater than one, the deposition site is selected at random. Otherwise, in the absence of this type of site, one proceeds as before but now considering sites implying two nearest-neighbour bonds and, finally, sites involving a single nearest-neighbour bond.

(b) Alternatively, one assumes that the development (100) \# is determined by the number of next-nearestneighbour bonds (diagonal bonds). That is, the corresponding rule is the same as has been already established for developing the (110) \# except that now the nearest-neighbour bond must be replaced by the next-nearest-neighbour bond. Likewise, it should be kept in mind that at least one nearest-neighbour bond must be formed in order to be consistent with the definition of the electrode profile (Fig. 2).

Let us stress that the two rules used to determine the most stable site for particle attachment are symmetrically equivalent; that is, the nearest-neighbour sites in (110) \# symmetry play the same role as the diagonal sites in (100) \# symmetry (Fig. 2). Furthermore, the dissolution rule is the same in both cases and in most simulations the values $p_{1}=p_{2}=0.2$ have been employed. Obviously, the preceding rules are the simplest ones to be used in Monte Carlo simulation. Certainly, more complicated rules could be set possibly providing a similar, or even a better, simulation of the electrochemical facetting mechanism of fcc metals. However, at the present stage a further extension in this direction is fully justified only after a relatively successful simple approach has been accomplished. It should be mentioned that other alternative rules have also been checked. For example, a model for developing preferred orientations exclusively based on the electrodissolution process gave unsatisfactory results.

The rules selected for the prevailing type of profile are fundamentally determined by the values of upper

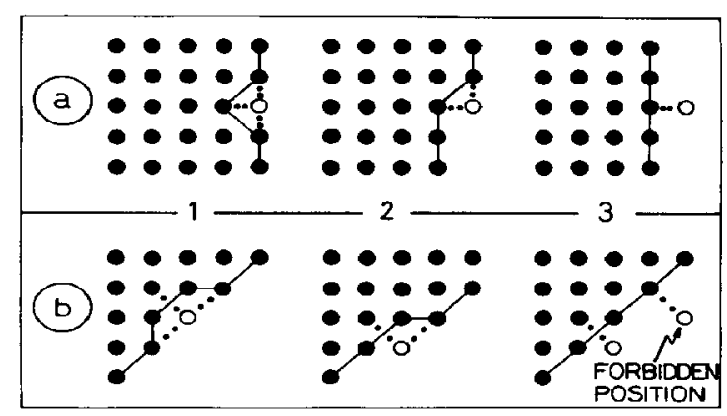

Fig. 2. Rules for the deposition of particles leading to the development of $(110)$ \# (a) and (100) \# profiles (b). The figures show the symmetry of the rules for some typical cases. The deposited particles has 3 nearest-neighbour (diagonals) for case 1a (1b) which corresponds to the filling of a vacancy; 2 nearest-neighbour (diagonals) in the case $2 \mathrm{a}$ (2b), which corresponds to step grow; and $1 n-n$ (diagonal) in the case $3 a$ (3b) which represents the adsorption of an atom on a perfect plane. For case $3 b$ a particle with only one diagonal bond and no nearest-neighbour bond is shown. The deposition of this particle is forbidden. 
and lower potential limits fixed for the periodic squarewave potential applied to the electrode. In this respect it should be noticed that the active electrochemical facetting of platinum in acids $[1-3,13,14]$, implies an upper potential more positive than the potential of the metal-metal ion reversible reaction. In this case, in the potential range of the upper potential limit the nonequilibrium adsorption conditions for anions apparently behaves as almost independent of the applied potential[15-18] and, the electrodissolution process appears to be independent of the choice of the different crystallographic faces of the metal. On the other hand, for the same example, potential values which are more negative than the reversible potential of the metal-metal ion reaction, determine the extent of reversible $\mathrm{H}$-adatoms formed on the electrode simultaneously with the metal-ion electrodeposition processes. Therefore, the final attachment of metal ions at the surface yielding faces of different atomic crystallographic packing depends whether the process occurs in the presence or in the absence of $\mathbf{H}$-adatoms.

\section{RESULTS AND DISCUSSION}

The Monte Carlo simulation was made by starting from different profiles representing either an arbitrarily rough profile, an ideal single crystal profile or a sawtooth profile. To facilitate the comparison between the different examples treated in this work, Table 1 summarizes the parameters employed in the Monte Carlo simulations as well as the relevant results obtained. It must be noticed that, when computing the number of particles lying in a given profile, only lines defined at least by three particles have been considered (Fig. 1).

The first example attempts to change a rough profile into a (100) \# profile (Fig. 3a and Table 1). During each dissolution half-cycle, a fraction $f=$ (number of detached particles $\left./ L_{2}\right) \cong 0.24$ of particles in the profile are typically detached from the electrode. After 60 Monte Carlo cycles, the profile exhibits a clear smoo- a

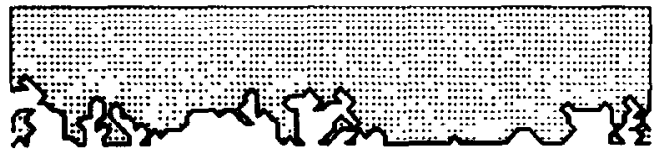

b

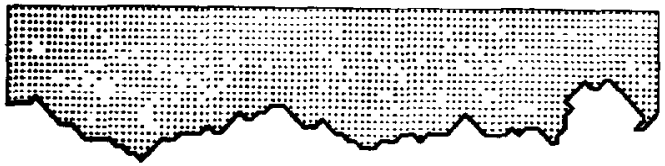

c

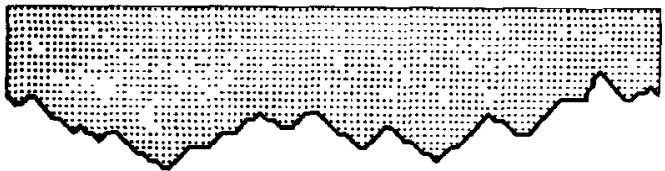

Fig. 3. Monte Carlo simulation for development of (100) \# profile starting from a rough profile (a) Starting electrode configuration; (b) Snapshot picture after 60 Monte Carlo cycles; (c) Snapshot picture after 130 Monte Carlo cycles. Simulation parameters and results are assembled in Table 1.

thing (Fig. 3b) and a large number of sites where the (110) \# profile is initiating (nucleation sites for preferred growth). At this stage the real profile length and correspondingly its roughness become smaller than those for the starting electrode. Small peninsulas and bays with preferred orientation are also formed. But after 130 Monte Carlo cycles (Fig. 3c) $f$ has diminished slightly to $f \cong 0.20$. Furthermore, about $64 \%$ of the total number of particles in the electrode profile lie in the $(100)$ \# profile and the electrode roughness becomes rather low, $S_{R} \cong 1.3$.

Another simulation was made by setting the rules corresponding to the development of a (110) \# profile

Table 1. Parameters employed in the examples depicted in Figs 3-7 and results from Monte Carlo simulation. $L_{1}=51, L_{3}=21, C=1.0$ and $p_{1}=p_{2}=0.2$. For all cases $L_{2}=101$, except in Fig. 6 where $L_{2}=96$. (SE) $=$ starting electrode.

\begin{tabular}{|c|c|c|c|c|c|c|c|}
\hline $\begin{array}{l}\text { Figure } \\
\text { number }\end{array}$ & $p$ & $L_{D}$ & $\begin{array}{l}\text { Monte } \\
\text { Carlo } \\
\text { cycles }\end{array}$ & $s_{R}$ & $\begin{array}{l}\% \text { of atoms } \\
\text { at }(100) \# \\
\text { profiles }\end{array}$ & $\begin{array}{c}\% \text { of atoms } \\
\text { at }(110) \# \\
\text { profiles }\end{array}$ & $\begin{array}{c}\text { Profile change } \\
\text { due to Monte } \\
\text { Carlo simulation } \\
\text { rules }\end{array}$ \\
\hline $3 a$ & 0.6 & - & $0 .(\mathrm{SE})$ & 2.5 & 19 & 40 & rough \\
\hline $3 b$ & & 10 & 60 & 1.5 & 41 & 39 & \\
\hline $3 c$ & & 10 & 130 & 1.3 & 64 & 23 & $(100) \#$ \\
\hline $4 a$ & 0.6 & - & $0 .(\mathrm{SE})$ & 3.0 & 37 & 38 & rough \\
\hline $4 b$ & & 10 & 10 & 1.8 & 12 & 66 & \\
\hline $4 c$ & & 10 & & 1.2 & 8 & 87 & (110)\# \\
\hline $5 \mathrm{a}$ & 1.0 & - & $0 .(\mathrm{SE})$ & 1.0 & 0 & 100 & (110)\# \\
\hline $5 b$ & & 10 & 60 & 1.2 & 32 & 31 & \\
\hline $5 c$ & & 10 & 200 & 1.3 & 50 & 40 & $(100) \#$ \\
\hline $6 a$ & - & - & 0.(SE) & 1.4 & 100 & 0 & $(100) \#$ \\
\hline $6 \mathrm{~b}$ & & 10 & 50 & 1.3 & 22 & 62 & \\
\hline $6 c$ & & 10 & 160 & 1.2 & 6 & 80 & $(110) \#$ \\
\hline $7 a$ & 0.8 & - & $0 .(\mathrm{SE})$ & 1.1 & 0 & 83 & rough \\
\hline $7 b$ & & 1 & 120 & 2.2 & 27 & 27 & \\
\hline $7 c$ & & 10 & 120 & 1.3 & 50 & 20 & $(100) \#$ \\
\hline
\end{tabular}


(Fig. 4 and Table 1). As the previous case, it should be noted that after a number of Monte Carlo cycles (roughly 80-130 depending on the profile one at tempts to develop) only minor changes in the electrode profile can be observed. This result is a consequence of both the small fraction of particles involved in each cycle and the development of a compact (void-free) preferred, oriented layer which penetrates some distance into the electrode, (void-free space).

The third example concerns the development of a (10) \# profile starting from a (110) \# profile (Fig. 5

a

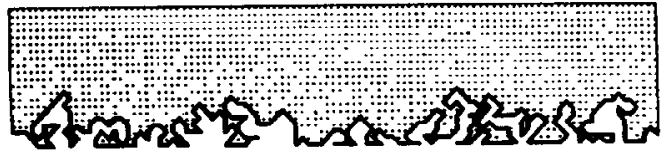

b

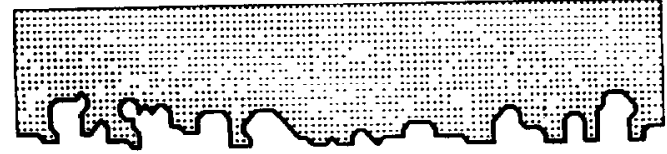

c

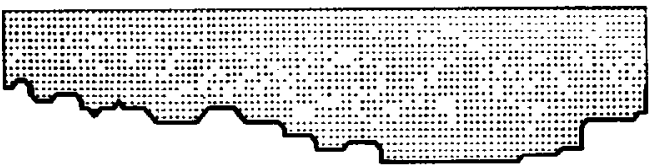

Fig. 4. Monte Carlo simulation for development of (110)\# profiles starting from a rough profile. The number of the Monte Carlo cycles in each case, simulation parameters and results are given in Table 1.

a

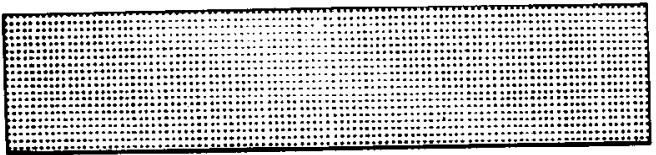

b
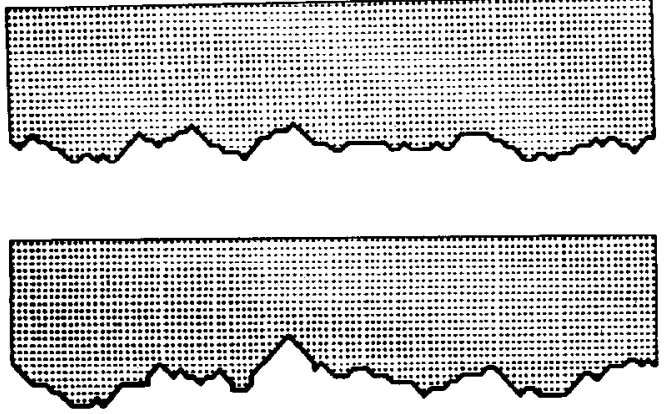

Fig. 5. Monte Carlo simulation for development of (100) \# profiles starting from a (110) \# profile. The corresponding numerical data are presented in Table 1. and Table 1). The sequence of configurations appearing during the Monte Carlo cycling is comparable to that already described for Fig. 3, except that in this case no initial smoothing is noticed as a consequence of the compactness of the starting electrode, but there is a slight increase in the roughness of the profile. As in other cases a preferred, oriented profile with a number of steps is finally produced. Conversely to the case of the rough starting electrode, the absence of structural defects in this example makes the penetration of the particle arrangement more difficult and a greater number of Monte Carlo cycles is therefore required to obtain a well-oriented profile. Based on these results one can speculate about the role of intercrystalline gaps and defects as preferential sites for the electrodissolution process which on the one hand can supply ions to the growing, oriented surface while on the other hand they can facilitate the penetration effect.

The same topographical changes also result by treating a periodic (100) \# profile. This profile turns into a (110) \# profile (Fig. 6 and Table 1) by applying the proper rules.

Let us now discuss the effect of the diffusion length $\left(L_{D}\right)$ on the resulting electrode structure. By starting from the same initial configuration (Fig. 7a, Table 1) and by using the rule for the growth of $(100)$ \# the results for $L_{D}=1$ and $L_{D}=10$ are depicted in Figs $7 \mathrm{~b}$ and $7 c$, respectively. Similar configurations as those shown in Fig. $7 b$ but with different preferential orientation, have been observed by using the rule for the development of the (110) \# profile. Therefore, one can conclude that the final structure strongly depends on $L_{D}$. For a very small diffusion length, ie when the particle has little chance of finding the most stable position, the growth of a rough, slightly preferredoriented profile is observed.

\section{COMPARISON BETWEEN THE MODEL AND EXPERIMENTAL FACTS}

The Monte Carlo simulation for development of profiles with a preferred orientation offers a satisfactory qualitative correlation with most of the experimental observations reported for the electrochemical facetting of various fcc metals, for $L_{2}=101 ; L_{3}=21$ or $41 ; 0.7 \leqslant p \leqslant 1.0 ; L_{D} \leqslant 10 ; 0.10 \leqslant p_{1} \approx p_{2} \leqslant 0.20$ and $C=1$. The points of qualitative coincidence can be summarized as follows.

1. Selective particle detachment and deposition are basic processes which account for the development of preferred-oriented profiles provided that $L_{D}$ is sufficiently large, $L_{D}>3$. For example, for $L_{D} \leqslant 3$, rough profiles are obtained independently of the rule used. In the experiments of electrochemical facetting the triggering reaction is the fast electroadsorption of $\mathrm{OH}$ from underpotential discharge of water $[4,13,14]$ and the thickness of the pulsating diffusion-boundary layer can be related to the surface-diffusion distance, $L_{D}$, of electrodepositing ions. This magnitude is determined by the frequency of the periodic potential.

2. For rough starting profiles the early stage of the process implies a smoothing and development of growing centres for preferred orientation of attached of particles. This conclusion agrees with scanning tunneling microscope images of preferred-oriented 


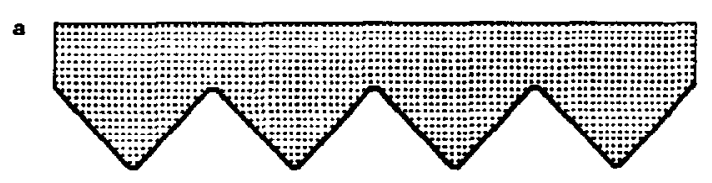

b
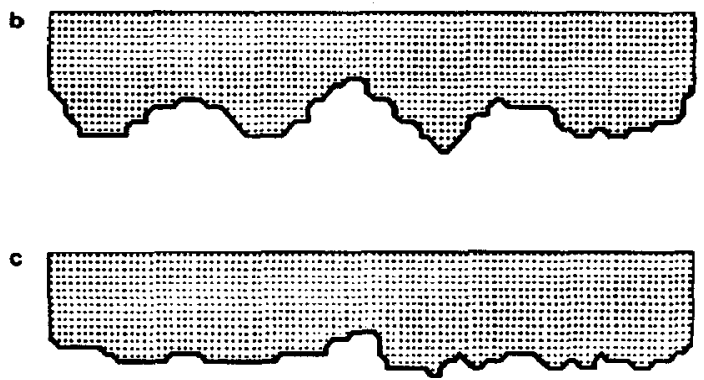

Fig. 6. Monte Carlo simulation for development of (110) \# profiles starting a (100) \# profile. The corresponding numerical data are presented in Table 1.

$\mathbf{a}$

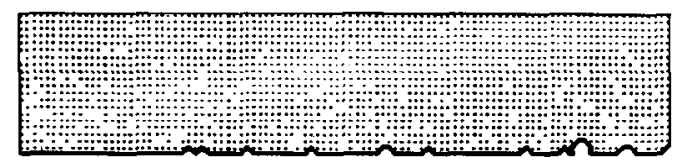

b

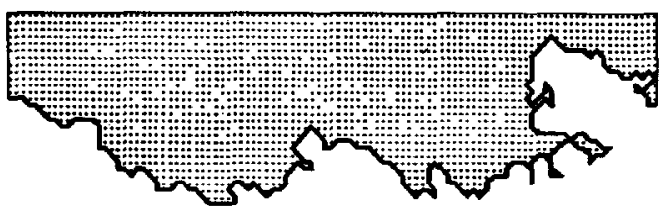

$c$

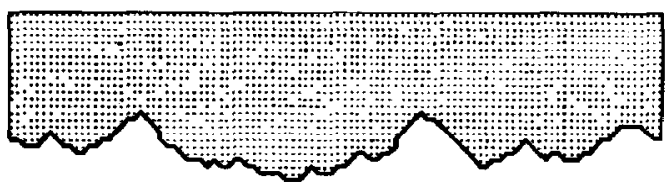

Fig. 7. The influcnce of the diffusion length on the profile resulting from Monte Carlo simulation (a) Initial starting electrode configuration; (b) $L_{D}=1,120$ Monte Carlo cycles; (c) $L_{D}=10,120$ Monte Carlo cycles. The rules for the development of $(100) \#$ profiles have been applied. The simulation parameters and the results are listed in Table 1.

platinum surfaces obtained for relatively short electrochemical facetting times $[4,19,20]$.

3. According to the model the type of preferred orientation depends on the deposition rule, ie on the lower potential limit of the periodic perturbation, but is independent of the upper potential limit, ie on the dissolution process. This conclusion correlates with the experimental fact that preferred orientation developed through electrochemical facetting depends upon the $\mathrm{H}$-adatom, adsorption-electroadsorption process coupled to the metal electrodeposition reaction $[4,13,14]$.

4. After a large number of Monte Carlo cycles one obtains: (a) an appreciable change in the relative distribution of crystallographic profiles with respect to the starting electrode configuration; (b) a stabilized step-like profile structure with preferred-oriented lines. After that, only a slight change in roughness is found in additional Monte Carlo cycles. The last statement and conclusion (a) agree with the electrochemical data obtained for $\mathrm{Pt}(111)$ preferred orientation $[1,2,4,15]$ Furthermore, the conclusion (b) is supported by scanning tunneling microscope images $[19,20]$ and scanning electron micrographies [21].

5. The results from Monte Carlo simulation indicate that the preferred orientation of particle arrangement penetrates into the electrode by several nearest-neighbour distances. The lack of voids in this layer is consistent with the experimental fact that the actual electrofacetted surfaces of fcc metals are very stable. In summary, conclusions 4 and 5 indicate that electrochemical facetting involves substantial changes of both the morphology and the topography of the electrode.

6. The final profile resulting from the model is independent of the initial starting electrode configuration. This conclusion agrees with electrochemical facetting results obtained for polycrystalline $[1-4,19]$ and single-crystal foc metals[22].

7. The development of the (110) \# profile is "easier" than the (100) \# profile, because in the former case fewer Monte Carlo cycles are necessary to obtain the stabilized profile. This conclusion is comparable with the fact that different potential-cycling times are required for the actual development of the different crystallographic orientations in fec metals $[4,14]$.

8. In the same sense as indicated in 7 , the development of the preferred oriented profile from a rough initial profile is easier than from a perfect profile. This conclusion also correlates with experimental electrochemical facetting data derived for both single[22] and polycrystalline $\operatorname{Pt}[1-4,19]$ by using square-wave potential perturbations.

In our opinion the Monte Carlo simulation method applied to the reaction model, although the latter can be further improved, brings forth at this stage a reasonable explanation for electrochemical facetting.

Acknowledgement-Financial support from the Consejo Nacional de Investigaciones Cientificas y Técnicas de la Republica Argentina, and the Comisión de Investigaciones Cientificas de la Provincia de Buenos Aires is acknowledged. We thank Alberto Maltz (CESPI, Universidad Nacional de La Plata) for his assistance in the development of computer programs.

\section{REFERENCES}

1. J. C. Canullo, W. E. Triaca and A. J. Arvia, J. electroanal. Chem. 175, 337 (1984).

2. R. M. Cerviño, W. E. Triaca and A. J. Arvia, J. electrochem. Soc. 132, 266 (1985).

3. C. L. Perdriel, W. E. Triaca and A. J. Arvia, J. electroanal Chem. 205, 279 (1986).

4. A.J. Arvia, J. C. Canullo, E. Custidiano, C. L. Perdriel and W. E. Triaca, Electrochim. Acta 31, (1986). 
5. U. Bertocci, Surf. Sci,; 15, 289 (1969); J. electrochem. Soc. 119,822 (1972).

6. J. Harrison and S. Rangarajan, Faraday Disc. Chem. Soc., 12, 70 (1978).

7. R. Armstrong and S. Church house, J. electroanal. Chem. 167,265 (1984).

8. W. Obretenov, V. Bostanov, E. Budevski, R. G. Barradas and T. J. Van der Noot, Electrochim. Acta 31, 753 (1986).

9. A. M. Feltham and M. Spiro, Chem. Rev. 71, 177 (19/1).

10. T. Biegler, Aust. J. Chem. 26, 2567 (1973).

11. R. Woods, Electroanalytical Chemistry (Edited by A. J. Bard). Vol. 9. p. 1, M. Dekker. New York (1976).

12. A. E. Bolzan, M. E. Martins and A. J. Arvia, J. electroanal. Chem. 157, 339 (1983).

13. R. M. Cerviño, W. E. Triaca and A. J. Arvia, J. electroanal, Chem. 182, 51 (1985).

14. W. E. Triaca, T. Kessler, J. C. Canullo and A. J. Arvia, J. electrochem. Soc. 134, 1165 (1987).
15. I. I. Labkovskaya, V. I. Kuk'yanycheva and V. S. Bagotskii, Sov. electrochem. 5, 535 (1969).

16. V. S. Bagotskii, Yu. B. Vassilyev, J. Weber and J. N. Pirtskhalava, J. electroanal. Chem. 27, 31 (1970).

17. H. Angerstein-Koslowska, B. E. Conway, B. Barnett and J. Mozota, J. electroanal. Chem. 100, 417 (1979).

18. F. E. Woodard, C. L. Scortichini and C. N. Reilly, $J$. electroanal. Chem. 151, 109 (1983).

19. J. Gōmez, L. Vāzquez, A. M. Barō, N. Garcia, C. L. Perdriel, W. E. Triaca and A. J. Arvia, Nature 323, 612 (1986).

20. L. Vázquez. J. M. Gómez R.. J. Gómez H., A. M. Baró. N García, J. C. Canullo and A. J. Arvia, Surf. Sci. 181, 98 (1987).

21. R. M. Cervin̄o, A. J. Arvia and W. Vieltich, Surf. Sci. 154, 623 (1985).

22. J. C. Canullo, W. E. Triaca and A. J. Arvia, J. electroanal. Chem. 200, 397 (1986). 